\title{
Exemplars can Reciprocate Principal Components
}

\author{
KIERAN GREER, \\ Distributed Computing Systems, Belfast, UK. \\ http://distributedcomputingsystems.co.uk
}

\begin{abstract}
This paper presents a clustering algorithm that is an extension of the Category Trees algorithm. Category Trees is a clustering method that creates tree structures that branch on category type and not feature. The development in this paper is to consider a secondary order of clustering that is not the category to which the data row belongs, but the tree, representing a single classifier, that it is eventually clustered with. Each tree branches to store subsets of other categories, but the rows in those subsets may also be related. This paper is therefore concerned with looking at that second level of clustering between the category subsets, to try to determine if there is any consistency over it. It is argued that Principal Components may be a related and reciprocal type of structure, and there is an even bigger question about the relation between exemplars and principal components, in general. The theory is demonstrated using the Portugal Forest Fires dataset as a case study. The Category Trees are then combined with other Self-Organising algorithms from the author and it is suggested that they all belong to the same family type, which is an Entropy-style of classifier. Some analysis of classifier types is also presented.
\end{abstract}

Key-Words: Cluster, Exemplar, Principal Component, Category Tree, Classifier Structure, Entropy.

Received: February 2, 2019. Revised: March 30, 2021. Accepted: April 13, 2021. Published: April 25, 2021.

\section{Introduction}

This paper presents a clustering algorithm that is an extension of the Category Trees [1] algorithm. Category Trees is a clustering method that creates tree structures that branch on category type and not feature. The development in this paper is to consider a secondary order of clustering that is not the category to which the data row belongs, but the tree, representing a single classifier, that it is eventually clustered with. Each tree branches to store subsets of other categories, but the rows in those subsets may also be related. This paper is therefore concerned with looking at that second level of clustering between the category subsets, to try to determine if there is any consistency over it. The mathematics for this problem could get quite complex and it will be argued that Principal Components [2] may be a related and reciprocal type of structure. This therefore poses an even bigger question about the relation between exemplars and principal components, in general. However, only a lightweight analysis of the mathematical properties is possible, really at the level of matching the different ingredients that are involved. The success criterion can also be decided in an iterative manner and may be the resulting clustered column with the lowest variance, or a lower variance compared to other results.

A second development is the decision that a number of classifiers created by the author in fact belong to the same family type, which is an entropystyle of classifier. They can even be combined to produce small improvements, where tests on a set of benchmark datasets demonstrate this and the properties that make them similar are described.

The problem was formulated when considering geographical data that might change over time. It is therefore a time-series problem, but one where the data is placed into discrete time bands and is not continuous. The distributed nature of the input suggested that a category could be created from each input station, or sensor set. This is a bit unusual, because the classifier would then be expected to cluster with itself, but the problem was expanded to not consider this aspect, but the aspect of how the data rows in each individual tree and its branches may be related. The branches represent subsets of data rows for other categories, where the original idea was that comparing these subsets for feature analysis would be more accurate than looking at the whole dataset. With time series, it would also be possible to consider how the relation changes over time. In fact, that is a more advanced problem than what has been concluded for this paper, where this test case considers only the relation between all rows in the branches together. The theory was tested with the forest fires dataset [3] as a specific case and to verify the result, the smaller El Nino dataset [4][5] has also been looked at.

The rest of this paper is organised as follows: section 2 describes some related work, while section 3 summarises the Category Trees and the new methods. Section 4 describes the environmental case study and gives a result that might help when 
monitoring forest fires. Section 5 discusses theoretical aspects, including principal components, and also presents test results for combining a set of the author's own classifiers. Section 6 describes the properties of a family type of classifier that they belong to, while section 7 gives some conclusions and possibilities for future work.

\section{Related Work}

This paper has become an extension to an earlier paper [6] that presented a self-organising algorithm and it will be argued that Concept Trees [7][8] and even the Frequency Grid [9] are similar types of entropy-based classifier. With regard to the selforganising algorithm, Adaptive Resonance Theory (ART) networks [10] were thought to be a close match and the Category Trees operate like Decision Trees [11][12][13]. The theory may have overlap with Principal Component Analysis (PCA) [2] and even the work of Oja [14] and others, who created neural network systems from these features. While principal components were learned, the problem reduced to a minimum when the features became similar, like a regression problem. It is interesting that Category Trees originated from trying to oscillate an error around a mean-centred value, created from a wave shape [15] representing the input data rows. That then became simply adjusting to the averaged batch row value. More specifically, similar ideas could be:

- Zero-mean could relate to the original oscillating error. It was found however that if dealing with a single averaged batch value, a single adjustment would suffice instead.

- Then of course the averaged values themselves and the closest fit to it. Not to realise maximum difference but to align the data rows with maximum similarity.

- Variance was also a part of the theory for a new entropy equation [16] that is intended to give some measure of cohesion across a pattern. It can also measure the cohesion across a tree structure, with possibly arbitrary concepts in the branches.

Using batch or averaged values is not new [17][18] and dates back to the early days of neural networks [19]-[21]. Measuring a juxtaposition between exemplars and principal components is unusual, when principal components are more often used as exemplars. Measuring the change in the variance is possibly more associated with KullbackLeibler divergence [22] and Information Gain theory [23]. As described in Wikipedia ${ }^{1}$, the KullbackLeibler divergence, (also called relative entropy), is a measure of how one probability distribution is different from a second, reference probability distribution. Entropy is represented in this paper by the variance. The reference distribution is then the original category exemplar and the new distribution is the exemplar after the data rows are re-clustered. Information Gain is then a method for measuring the entropy difference over different sets of variables. It does this by determining if the data can be split into certain subsets that may hold more consistent information than the whole dataset together. If the sum of the variances in the subsets is less than for the whole dataset, then that difference is the information gain, and it is also a reduction in the variance.

\section{Category Trees}

This section is a review of the Category Trees classifier [1]. The method is supervised, where each actual output category is assigned a classifier and the classifier learns to adjust from an averaged batch row value to the desired output value. This adjustment can in fact be done in a single step. Because each category is separate, it is like a neural network with a separate neuron for each output category and the value can be anything, but would typically be 1 or 0.5 , for example. The classifier weight set therefore adjusts from the averaged batch row value to the value 0.5 or 1 . Because the adjustment is for the averaged batch value, individual data rows may be closer to other classifier results. Therefore, after learning this adjustment, each data row is passed through each classifier again, and it is stored with the classifier that produces the smallest error with it. If the data row's category is not the same as the classifier, then a new layer is created in the classifier and the data row is stored with a new classifier in the new layer that represents the new category. The difference being that subsequent layers would be trained on smaller and smaller subsets of data rows and the base classifier is always representative of the original category. Then to retrieve the category information, a data row is passed to each classifier and the one with the smallest error in the base layer is used. If it

\footnotetext{
$1 \quad$ https://en.wikipedia.org/wiki/KullbackLeibler_divergence
} 
has branches, then the row is passed to each branch and the one with the smallest error again used, until the process terminates at a leaf node. The category of that leaf node is then declared to be the category for the data row.

The process is illustrated in Figure 1. The dataset contains two categories - A and B. Two classifiers are thus trained, one to recognise the averaged category A value and one for the averaged category $B$ value. When each individual row is then passed through the classifiers, some of the B category rows are closer to the averaged category A value. Therefore, the A classifier branches to a new layer, where it recognises both its own category A rows and a subset of the category $B$ rows. While this process should be very accurate, it is quite a shallow architecture and it may not generalise as well as it is able to learn the original data, but definitely still well-enough to be useful. The earlier paper [1] presented some ground-breaking results.
Classifier A incorrectly classifies Part of category B as closest

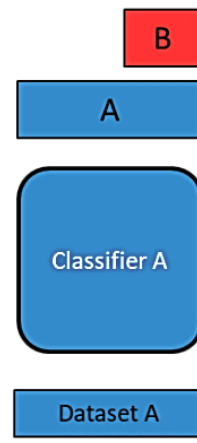

Dataset B

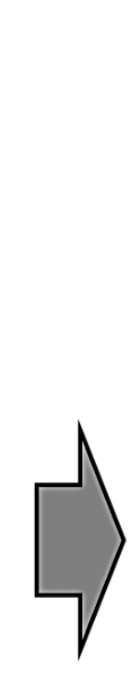

Phase 1 New layer added to classifier A which
now also classifies part of category B

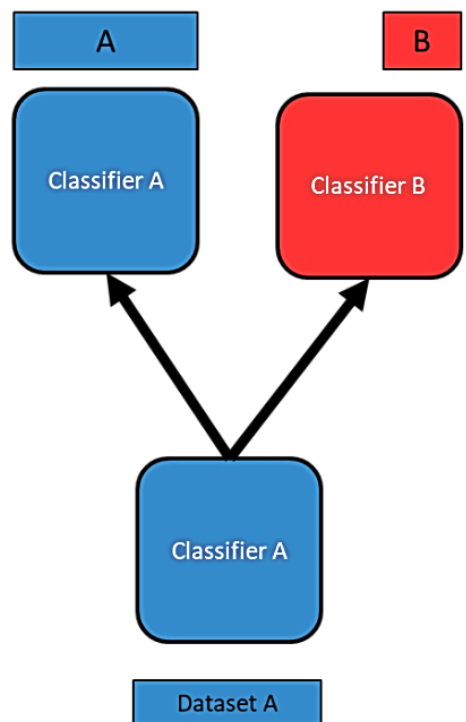

Phase 2
Now 3 potential

endpoints to determine the

classification from

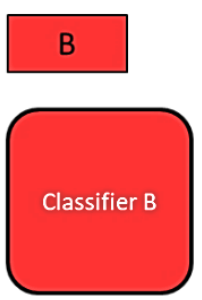

Dataset B

Figure 1. Schematic of the classifier in action. Phase 1 realises that classifier A also classifies part of category B better. Phase 2 then adds a new layer to classifier A, to re-classify this subset only.

\subsection{Secondary Clustering}

While each category can be distributed across any number of classifiers, there may also be the option to look at all of the data rows associated with each classifier tree separately. There is obviously some type of association there, because the data rows are closest to the classifier's representing value and so their feature sets must also be close. And because it is for subsets of rows, the comparison should be more accurate than if the feature sets of the whole dataset were compared. If 10 data rows from category B are clustered through the category A classifier, for example, then the averaged feature set for category B when it is similar to category A, can be from those 10 rows only. This is one possibility that would work with a supervised approach, when the categories are known and the representing value would probably also be a centroid. The Iris dataset [24], for example, is perfectly centered, with only 10 data rows clustered with other categories. Section 4 describes another possibility that may be useful when the representing value is an exemplar, but not a centroid. In that case, it represents something, but it may not be at the center of the category.

\subsection{Recursive Clustering}

As with entropy classifiers in general, such as Information Gain [23], a decision can be made to split a dataset on a set of variables that would 
produce a better entropy for the split parts. Deciding on the variable set can be arbitrary and iterative. Thus, there are different representations for a good category set and adjusting the result from an educated guess could be an option. If the selected category groups do not produce centroids, then individual data rows may be clustered with classifiers, other than their own base category, but further clustering of each classifier using the new sets of data rows, may continue to refine the exemplar.

Quite simply, when the rows are re-clustered with the closest exemplar, the process can be repeated by creating new exemplars from the new row batches and then re-clustering on those again. This can repeat until a minimum number of changes occur. If the process is supervised, with known category groups, then this should not improve the clustering performance by very much, but it may benefit an unsupervised or a semi-supervised procedure more. This may also mean that if it is better for the environmental scenario, then the distributed stations are semi-supervised at best and can gain knowledge through the secondary clustering. This also means that using Category Trees in a supervised environment would be an upper bound on how well it can classify a problem. Any skew can be learned directly by the classifiers. If instead, the recursive clustering can adjust the exemplars to a slightly different orientation, then the hope would be that an unsupervised environment that cannot inherently learn the skew, would benefit from the adjustment more, and it would help to align any guessed category set with the real one.

\section{$4 \quad$ Forest Fires Case Study}

The forest fires dataset [3] gives readings for a year, from Portugal's Monteshino natural park in the north east of Portugal. This was actually the first dataset that was tested, when it became clear that larger datasets could not be processed easily, but it is unlikely that the solution is specific to this one dataset only. The idea is to create categories based on the distributed nature of the input, where the dataset is divided into a 9x9 matrix and each location can represent a category. The fires that occurred in each region over the period of a year were recorded with the month and day of the fire and some sensor indicators. The weather sensors recorded temperature, relative humidity, wind speed and rain. As well as this, the fire indicators of Fine Fuel Moisture Code (FFMC), Duff Moisture Code (DMC), Drought Code (DC) and Initial Spread Index (ISI) were recorded with each weather reading. The original paper tried to predict when fires might occur and was more successful with the small fires. This paper suggests some type of relation between the location cells, where if there is a fire in one grid location, then other locations might also be vulnerable. The output criterion was taken to be the month that the fire occurred in and in fact there was a clear correlation there in the results. But there was also a large spread of related grid cells and so it might not be very accurate for predicting exactly when a fire would take place.

Deciding to convert the sectors into classifiers is an arbitrary decision, albeit with some intuition. Then there is also the output criterion, which has been selected to be the month of the year. This again is arbitrary and not implicit in the data, apart from the fact that it is what is being measured. Then a subset of the other variables was used to realise the relation between these two variables. The dataset was therefore divided into baches of rows, one batch for each grid location that had fires. The classifier for that location learned the average input value and used that as an exemplar for the location. After learning the averaged value, each row was presented to each classifier and it was clustered with the one it was closest to. That produced a new set of row batches for each classifier. The month for each row in each batch was then analysed and its variance was calculated. The average variance for the rows clustered for the classifiers was compared to the variance for each grid cell without clustering and the result is shown in Table 1.

\begin{tabular}{|c|c|}
\hline & Month of the Fire \\
\hline Variance Before & 1.32 \\
\hline Variance After & 0.35 \\
\hline
\end{tabular}

Table 1. Variance values for each Forest Fires Month variable before and after the clustering.

The surprising result was that the classifiers aligned the rows based on the month of the fire. A description of some of the clustered rows in given in Appendix A. Further analysis showed that a classifier might not have any of its own rows clustered with it, so while the average value represents the batch set of rows, no individual row from that batch might be clustered with the classifier. As the first two clusters in Appendix A show, there are no data rows from the sector finally clustered with it. This could suggest that the clustering criterion is not distance-based. The classifier therefore does not produce a centroid, at 
the centre of the category, but some other representative value. This is explained further in section 5. The environmental result however, would be that because the data is clustered consistently on the month, it could help a fire service to monitor the region, as it could follow the sequences of locations when any fires started in one of them.

\subsection{El Nino Dataset}

The smaller El Nino dataset [4][5] was also tested. Any row with a missing value was removed first, resulting in 507 rows and then each buoy was allocated to be a category. This also related to a specific location and then columns 4 to 8 were used to train the classifier. The variance of each of these columns was then compared before and after the training, for each category, where the result is shown in Table 2. The re-clustering of the rows has therefore produced a smaller variance in 4 of the 5 variables and an almost equal variance in the fifth one. This is slightly different to the forest fires dataset, when the output variable was not included in the training rows.

\begin{tabular}{|l|l|l|l|l|l|}
\hline & $\begin{array}{l}\text { Zon. } \\
\text { Wind }\end{array}$ & $\begin{array}{l}\text { Mer. } \\
\text { Wind }\end{array}$ & Humidity & $\begin{array}{l}\text { Air } \\
\text { Temp. }\end{array}$ & $\begin{array}{l}\text { S.S } \\
\text { Temp. }\end{array}$ \\
\hline $\begin{array}{l}\text { Variance } \\
\text { Before }\end{array}$ & 1.15 & 1.1 & 2.1 & 0.38 & 0.245 \\
\hline $\begin{array}{l}\text { Variance } \\
\text { After }\end{array}$ & 0.85 & 0.8 & 1.5 & 0.26 & 0.25 \\
\hline
\end{tabular}

Table 2. Variance values for each El Nino variable before and after the clustering.

\section{Category Trees as Principal Components}

The case study shows that a classifier may not end up with any of its own category rows clustered with it. If the relation was for centroids, then it would be expected that the rows for the category would be clustered with it and so possibly each classifier value is an exemplar instead. Therefore, the clustering is not based on distance alone and the use of exemplars might point to a principal component that is not aligned exactly with its input data, but with some other type of feature set.

Principal Component Analysis converts the data into vectors of maximum variance. The PCA vector lines replace the data rows and when learned can be used to discriminate where the dataset changes most. Principal components are statistically the most significant features. The exemplars of this paper would instead represent directions of minimum variance, or maximum similarity. While that similarity is defined by some arbitrary criterion, it may be possible to formulate this problem in a general sense. It could be imagined that in an unsupervised setting, where these variable sets are not known, some iterative process could try different combinations and maybe measure the variance over the output criteria, to select the best matching combinations with the lowest variances.

The following section does not test the iterative process, but adds the category trees to some of the author's own algorithms and repeats some earlier tests for comparison.

\subsection{Tests Over Benchmark Datasets}

The test scenario is a repeat of the tests carried out in [6], which reported results for a new selforganising algorithm. A computer program has been written in the $\mathrm{C \#}$ dotnet language, by the author for his own algorithms and used to test some benchmark datasets that can all be found in the UCI Machine Learning repository [25]. The data was presented with the category information removed and also with the row order randomised. The only information the self-organising algorithm used to start with was the distance between each of the data rows. From that, it created initial sets of clusters based on closest distances, using a full-linking and cross-referencing method that is described in [6]. It is also an agglomerative method, where the stopping criterion used was the cluster set just before the actual number of clusters fell below the desired number. This probably results in a bias towards a larger number of clusters in the result. The strength of the algorithm is that it can produce coherent cluster sets, meaning that the data rows in each cluster would all belong to the same actual category. It was then possible to calculate the error for this as follows:

1. For every sub-cluster, retrieve from the dataset, the category for each row.

2. Remove the set of rows with the largest count for a single category.

3. The coherence error is then the number of rows left.

So, for example, if a cluster set contains data rows for categories as follows: A, A, A, B, B, then there would be 2 incorrect nodes. If the dataset actual categories were: A, A, A, B, B, C, C, then the error would be 4 .

As stated in section 3.2, using Category Trees in a supervised environment would be an upper limit on the performance. The tests therefore, were for the self-organising algorithm, but then compared to new 
versions that also include the Category Trees classifier. The self-organising algorithm uses a closest distance measure to cluster individual nodes and then a new Frequency Grid [9] to further cluster those events. Because the supervised Category Trees can learn an inherent skew and even re-align to a different set of vectors, using the unknown results of the self-organising algorithm as the base cluster set for the trees may help to re-align some of those data rows again, to produce a more correct orientation. Then there is also the idea of recursively feeding the Category Trees result back to itself. In this case, only 1 recursive feedback was used. The results of using these 3 algorithms are given in Table 3, where:

- $\mathrm{SO}$ is the self-organising algorithm by itself.

- SO-CT is the self-organising algorithm, that feeds into standard category trees, that feed into the next self-organising level.

- SO-SRCT is the self-organising algorithm, that feeds into category trees that use the secondary ordering and 1 phase of recursion, before feeding back into the next self-organising level.
- Each cell score gives: the number of clusters first, then the percentage of cohesion, as described above, and then the frequency grid version that produced the result.

- The average cluster error is the number above the actual number of 3.7, and the average accuracy percentage is the cohesion accuracy.

The self-organising algorithm scores very well by itself, so there is not much room for improvement. Each test was run over 3 iterations instead of 10 and this produced better results than what was published in [6]. The results from 50 tests were then averaged to give the final score. The results for fewer iterations also showed a much more even split between version 1 or version 2 of the frequency grid. With relation to other heuristics, a value above $90 \%$ is considered to be acceptable for unsupervised clustering for the more separable datasets, such as the Iris dataset.

\begin{tabular}{|l|l|l|l|}
\hline & SO & SO-CT & SO-SRCT \\
\hline Iris (150-3) & $5-94 \%$ V1 & $7-93 \% \quad$ V1 & $4-91 \% \quad$ V1 \\
\hline Wine (178-3) & $8-91 \%$ V1 & $5-98 \%$ V1 & $9-95 \% \quad$ V1 \\
\hline Zoo (101-7) & $8-99 \%$ V2 & $15-98 \% \quad$ V2 & $15-98 \% \quad$ V2 \\
\hline Hayes-Roth (132-3) & $4-69 \%$ V1 & $4-71 \% \quad$ V1 & $4-77 \% \quad$ V2 \\
\hline Heart Disease Cleveland (303-5) & $7-77 \%$ V2 & $7-73 \%$ V2 & $13-80 \% \quad$ V1 \\
\hline Sonar (208-2) & $4-82 \%$ V2 & $3-88 \%$ V1 & $3-88 \% \quad$ V2 \\
\hline Wheat Seeds (210-3) & $7-88 \%$ V2 & $10-97 \%$ V1 & $8-97 \% \quad$ V1 \\
\hline Average Cluster Error & 2.44 & 3.7 & 4.3 \\
\hline Average Accuracy Percentage & $85.5 \%$ & $86.5 \%$ & $89.5 \%$ \\
\hline
\end{tabular}

Table 3. Test results on some benchmark datasets.

\subsection{Test Conclusions}

The results are very pleasing and are what the author would like to demonstrate. But the values can still change quite a lot and so while they are accurate and representative, they should also be taken with a pinch of salt. While the error is improved, the number of clusters also increases, which is not a good result, but not as serious as the error reduction. The idea is that the supervised Category Trees can introduce a skew to the self-organised clusters and that will help them to re-align to a more accurate set of exemplars. If the secondary clusters and recursive feedback also has an effect, then better still, because the first category tree learning phase can only learn the cluster set presented by the self-organising algorithm. The adjustment comes in the second recursive stage. Note however that if an optimum result is achieved, any further stage will move away from it and so only 2 stages were tried in the recursive procedure. Also, if the test dataset is properly formed, then the exemplars are also centroids and the secondary clusters would be almost the same.

Changing the number of test runs had a significant effect, where it was reduced from 10 to 3 in this set of tests. More significantly, the value was also used for the final ensemble loop, which is like a Random Forest [12][13]. The cluster ordering had to be randomised before this final frequency grid stage, because the ordering has an effect on the clusters that it produces, see [6], section 4.2.1. After the top counts that create the clusters however, there are a 
large number of lower counts that could produce a lot of overlap in the different ensemble sets. If the number of random sets is reduced, then the overlap is also less and this may help to keep the different category clusters separate. The frequency grid also produced clusters with a large variation of frequency counts in the first layer, but the counts became much more uniform in subsequent clustering layers. This may be why a smaller 'test runs' value gave a better performance, because it was also used to determine the number of ensemble cycles. Especially with a global optimum, the values can tend to the minimum value and then saturate, when they all receive a similar score. It can be better to stop the training before the saturation happens and use the more variable output, albeit with a slightly larger error value. Time is then a factor, where certain variables with a greater affinity to the minimum value, will get there first.

Overtraining is a well-known problem, but that is typically when comparing with a different test dataset and it usually still produces a better fit in the train dataset. In fact, the ensemble loop should probably be a separate value, so that this problem does not occur. New tests confirm that this correction would be an option. Concerning the preference for version 2 of the frequency grid, it produces larger clusters to start with and so it would probably work better with fewer ensemble aggregations afterwards. The classifier rather than the overlap determines the clusters.

\section{The Same Family of Classifiers}

A second tree structure suggested by the author is a Concept Tree [7][8]. This stores concepts of arbitrary nature, with the simple mathematical rule that a child node cannot have a larger frequency count than its parent. It is interesting that Category Trees also supports this rule. As classifiers in branches store only subsets of nodes, they are likely to reduce their number of rows each time, which is like reducing a frequency count. If the variance inside of each tree is less than for the global population, then that gives the desired cohesion that the Concept Tree requires. It may be the case that Category Trees, Concept Trees and even the Frequency Grid, belong to the same family of classifier. Category Trees are maybe a supervised version, where Concept Trees are semi-supervised or unsupervised. This family of classifier makes use of averaged or batch values instead of individual local values. It is concerned with reducing the entropy or variance of the structure, which is again a global error correction over smaller local corrections. Even the Frequency Grid clusters use the largest count from a global table and not local relations. It may be the case that the counting rule of the Concept Tree that also occurs in the Category Tree structure leads to global minima, compared to the much more diverse landscape that a neural network might produce [21]. Using this in conjunction with fewer links between nodes could also be a factor.

If the search space is a global optimum, even a relatively shallow one, then it makes sense that only one correction is required to move to the best result. It is clear that the nature of the classifier and even its structure, is related to the error surface topology that it produces. But it is not the case that all single-layer classifiers produce global optima, where feedback between nodes could be a factor. It is now easy to see however, the uneven error topology for a fully connected multi-layer neural network with different weight sets and so the number of dimensions in a move may be more significant. Hill-climbing, for example, only leads to local optima, but it only deals with local information. The new classifier family is able to obtain information at a global level and then each move is very low dimension, only 1 dimension or link really. The cost then is a reduction in the level of detail in the information.

This is also interesting, because in more abstract terms, operations for one of the classifiers can maybe be tried on the other one. The similarity becomes even more pronounced when both tree structures perform a slight adjustment, through an internal recursive update, as described here in section 3.2 and in the paper [8] section 7.3, for the Concept Trees. In that paper, it was like a matching process that refined itself, by a small amount.

\section{Conclusions}

This paper has introduced a new clustering method that can improve the variance for similarity, across the cluster sets. It is based on the Category Trees algorithm, which is a supervised approach, but the new method may eventually work in an unsupervised manner. The method creates exemplars instead of centroids and poses the question if exemplars and principal components are related in some way. Environmental data is used as a case study and the result could help the fire service to monitor a region for fires. The advantages are the high levels of accuracy and the simplicity of the design, compared to many levels and number of weights in neural networks, for example. A set of the author's algorithms have been placed into a family 
type that is an entropy-style of classifier. Test results on combinations of this family show improved results to what was published previously for the classifiers and also that the different classifiers can work well together.

\subsection{Future Work}

The author has an interest in building a brain-like model and he hopes to integrate the self-organising unit into that. The secondary clustering is interesting because it provides information that is not directly available and the process can possibly be automated. It results from Category Trees that branch on category type and not feature. Most classifiers branch on feature and so an investigation into whether this is a unique aspect of Category Trees would be one option.

Because the row numbers are reduced, this could lead to more accurate analysis of similarities or differences between row sets, clustered in the same classifier. Or between feature subsets and the exemplar itself. Principle components are directions of maximum variance over the features, while the new components are directions of maximum similarity, again over the features. This therefore indicates two bounds on the problem. Can they be used together and give an understanding to the space between them? If we have one set of these components, would that help to guess what the other set might be? One other direction of research may be to look more closely at the classifier structure and try to relate this with the error surface. Do monotonically decreasing or increasing counts across layers produce certain types of error surface, for example?

\section{References:}

[1] Greer, K. (2018). An Improved OscillatingError Classifier with Branching, WSEAS Transactions on Computer Research, Vol. 6, pp. 49 - 54. E-ISSN: 2415-1521. For the updated version, see Category Trees (2020), available on arXiv at https://arxiv.org/abs/1811.02617.

[2] Wold, S., Esbensen, K. and Geladi, P. (1987). Principal component analysis. Chemometrics and intelligent laboratory systems, Vol. 2, No. 1-3, pp. 37-52.

[3] Cortez, P. and Morais, A. (2007). A Data Mining Approach to Predict Forest Fires using Meteorological Data. In J. Neves, M. F. Santos and J. Machado Eds., New Trends in Artificial Intelligence, Proceedings of the 13th EPIA 2007 - Portuguese Conference on Artificial Intelligence, December, Guimaraes, Portugal, pp. 512-523. APPIA, ISBN-13 978-989-95618$0-9$.

[4] El Nino Dataset, http://archive.ics.uci.edu/ml/ datasets/El+Nino.

[5] Bay, S.D., Kibler, D.F., Pazzani, M.J. and Smyth, P. (2000). The UCI KDD Archive of Large Data Sets for Data Mining Research and Experimentation. SIGKDD Explorations, 2.

[6] Greer, K. (2020). A Pattern-Hierarchy Classifier for Reduced Teaching, WSEAS Transactions on Computers, ISSN / E-ISSN: 1109-2750 / 22242872, Volume 19, 2020, Art. \#23, pp. 183-193.

[7] Greer, K. (2014). Concept Trees: Building Dynamic Concepts from Semi-Structured Data using Nature-Inspired Methods, in: Q. Zhu, A.T Azar (eds.), Complex system modelling and control through intelligent soft computations, Studies in Fuzziness and Soft Computing, Springer-Verlag, Germany, Vol. 319, pp. 221 252, 2014.

[8] Greer, K. (2019), Adding context to Concept Trees, International Journal of Intelligent Systems Design and Computing, Inderscience, Vol. 3, No. 1, pp.84-100. DOI: 10.1504/IJISDC.2019.105805.

[9] Greer, K. (2019). New Ideas for Brain Modelling 3, Cognitive Systems Research, Vol. 55, pp. 1-13, Elsevier. DOI: https://doi.org/10.1016/j.cogsys.2018.12.016.

[10] Carpenter, G.A. and Grossberg, S., 2010. Adaptive resonance theory.

[11] Breiman, L., Friedman, J., Stone, C.J. and Olshen, R.A. (1984). Classification and regression trees. CRC press.

[12] Breiman, L. (2001). Random Forests. Machine Learning, Vol. 45, No. 1, pp. 5 - 32.

[13] Adnan, M.N. and Islam, M.Z. (2015). Improving the random forest algorithm by randomly varying the size of the bootstrap samples for low dimensional data sets. In Proceedings of the European Symposium on Artificial Neural Networks, Computational Intelligence and Machine Learning, pp. 391 396.

[14] Oja, E. (1982). A Simplified Neuron Model as a Principal Component Analyzer, J. Math. Biology, Vol. 15, pp. 267-273.

[15] Greer, K. (2013). Artificial Neuron Modelling Based on Wave Shape, BRAIN. Broad Research in Artificial Intelligence and Neuroscience, Vol. 4, Nos. 1-4, pp. 20-25, ISSN 2067-3957 (online), ISSN 2068 - 0473 (print).

[16] Greer, K. (2021). A Brain-like Cognitive Process with Shared Methods, Int. J. Advanced Intelligence Paradigms, Vol. 18, No. 4, pp.481- 
501, Inderscience, DOI: 10.1504/IJAIP.2018. 10033335.

[17] Gallant, S.I. (1990). Perceptron-Based Learning Algorithms, IEEE Transactions on Neural Networks, Vol. 1, No. 2.

[18] Hagan, M.T. and Menhaj, M.B. (1994). Training Feedforward Networks with the Marquardt Algorithm, IEEE Transactions on Neural Networks, Vol. 5, No. 6, pp. 989-993.

[19] Hinton, G.E., Osindero, S. and Teh, Y.-W. (2006). A fast learning algorithm for deep belief nets, Neural computation, Vol. 18, No. 7, pp. $1527-1554$.

[20] Rojas, R. (1996). Neural Networks: A Systematic Introduction. Springer-Verlag, Berlin and online at books.google.com.

[21] Widrow, B. and Lehr, M. (1990). 30 Years of adaptive neural networks: perceptron, Madaline and backpropagation, Proc IEEE, Vol. 78, No. 9, pp. 1415-1442.

[22] Kullback, S. and Leibler, R.A. (1951). On information and sufficiency, Annals of Mathematical Statistics, Vol. 22, No. 1. pp. 79 86. doi:10.1214/aoms/1177729694.

[23] Mladenic, D., Brank, J., Grobelnik, M. and Milic-Frayling, N. (2004). Feature Selection using Linear Classifier Weights: Interaction with Classification Models, In SIGIR, Sheffield, U.K, pp. 234-241.

[24] Fisher, R.A. (1936). The use of multiple measurements in taxonomic problems, Annual Eugenics, 7, Part II, pp. 179-188, also in Contributions to Mathematical Statistics (John Wiley, NY, 1950).

[25] UCI Machine Learning Repository (2021). http://archive.ics.uci.edu/ml/.

\section{Appendix A - Examples of the Forest Fires Clusters}

This appendix lists some of the clusters generated by the secondary ordering of the Category Trees classifier. There were several clusters for the August - September time period, where two are displayed first. Note that there are no rows from the $(1,2)$ sector in that cluster or from the $(1,3)$ sector in that cluster.

Rows clustered for Sector $(1,2)$

$\mathrm{X}$, Y, month, day, FFMC, DMC, DC, ISI, temp, $\mathrm{RH}$, wind, rain, area

2,4,aug,tue, $94.8,108.3,647.1,17,20.1,40,4,0,0$

2,4,aug,wed,92.1,111.2,654.1,9.6,20.5,35,4,0,1.64

4,3,aug,wed,92.1,111.2,654.1,9.6,20.4,42,4.9,0,0
4,3, aug,wed,92.1,111.2,654.1,9.6,20.4,42,4.9,0,0

6,3,aug,fri,91.1,141.1,629.1,7.1,19.3,39,3.6,0,1.56

6,4,aug,thu,95.2,131.7,578.8,10.4,20.3,41,4,0,1.9

Rows Clustered for Sector $(1,3)$

1,4,aug,wed,91.7,191.4,635.9,7.8,19.9,50,4,0,82.75

2,2 ,sep,fri, $92.4,117.9,668,12.2,23,37,4.5,0,0$

2,2,aug,tue, $92.1,152.6,658.2,14.3,21.8,56,3.1,0,0.52$

3,4 ,sep,sun,92.4,124.1,680.7,8.5,22.5,42,5.4,0,0

4,4,sep,sun, $93.5,149.3,728.6,8.1,22.9,39,4.9,0,48.55$

5,4 ,aug,tue, $88.8,147.3,614.5,9,17.3,43,4.5,0,0$

5,4 ,aug,tue, $95.1,141.3,605.8,17.7,24.1,43,6.3,0,2$

6,5 ,sep,fri, $93.3,141.2,713.9,13.9,22.9,44,5.4,0,0$

7,4,aug,sun,91.4,142.4,601.4,10.6,20.1,39,5.4,0,2.74

7,5,aug,tue, $96.1,181.1,671.2,14.3,21.6,65,4.9,0.8,0$

8,6,aug,tue, $92.1,152.6,658.2,14.3,20.1,58,4.5,0,9.27$

8,6 ,aug,tue, $96.1,181.1,671.2,14.3,21.6,65,4.9,0.8,0$

These were different cluster sets for the Winter period:

Rows Clustered for Sector $(4,6)$

3,5 , mar,mon, 87.6,52.2,103.8,5,9,49,2.2,0,0

4,5,jan,sun, $18.7,1.1,171.4,0,5.2,100,0.9,0,0$

4,6,dec,sun,84.4,27.2,353.5,6.8,4.8,57,8.5,0,8.98

4,6,dec,thu, $84.6,26.4,352,2,5.1,61,4.9,0,5.38$

4,6, dec,fri, $84.7,26.7,352.6,4.1,2.2,59,4.9,0,9.27$

6,3, feb,sun, $84.9,27.5,353.5,3.4,4.2,51,4,0,0$

6,3, nov,tue, $79.5,3,106.7,1.1,11.8,31,4.5,0,0$

6,5 ,jun,sat, $53.4,71,233.8,0.4,10.6,90,2.7,0,0$

8,6,dec,wed, $84,27.8,354.6,5.3,5.1,61,8,0,11.19$

Rows Clustered for Sector $(7,3)$

3,4, dec,mon, $85.4,25.4,349.7,2.6,4.6,21,8.5,0,10.73$

4,4, dec,mon, $85.4,25.4,349.7,2.6,4.6,21,8.5,0,17.85$

4,4, dec,mon, $85.4,25.4,349.7,2.6,4.6,21,8.5,0,22.03$

$4,4, \mathrm{dec}, \mathrm{mon}, 85.4,25.4,349.7,2.6,4.6,21,8.5,0,9.77$

6,5, dec,tue, $85.4,25.4,349.7,2.6,5.1,24,8.5,0,24.77$

\section{Creative Commons Attribution License 4.0 (Attribution 4.0 International, CC BY 4.0)}

This article is published under the terms of the Creative Commons Attribution License 4.0

https://creativecommons.org/licenses/by/4.0/deed.en_US 